\title{
High Growth Speed of Gallium Nitride Using ENABLE-MBE
}

J. J. Williams ${ }^{1}$, A. M. Fischer ${ }^{2}$, T. L. Williamson ${ }^{3}$, S. Gangam ${ }^{4}$, N.N. Faleev ${ }^{4}$, M.A. Hoffbauer ${ }^{2}$, C. B. Honsberg ${ }^{4}$

${ }^{1}$ Materials Science Engineering, Arizona State University, P.O. Box 875706, Tempe, AZ 852875706, USA

${ }^{2}$ Department of Physics, Arizona State University, P.O. Box 871504, Tempe, AZ 85287-1504, USA ${ }^{3}$ Chemistry Division, Los Alamos National Laboratory, P.O. Box 1663, MS J565, Los Alamos, NM 87545, USA

${ }^{4}$ School of Electrical, Computer and Energy Engineering, Ira A. Fulton Schools of Engineering, Solar Power Lab, Arizona State University, P.O. Box 875706, Tempe, AZ 85287-5706, USA

\begin{abstract}
Films of gallium nitride were grown at varying growth speeds, while all other major variables were held constant. Films were grown determine the material impact of the high flux capabilities of the unique nitrogen plasma source ENABLE. Growth rates ranged from 13 to near $60 \mathrm{~nm} / \mathrm{min}$. X-ray $\omega$ scans of GaN (0002) have FWHM in all samples less than 300 arcsec. Cathodoluminescence shows radiative recombination for all samples at the band edge. In general material quality overall is high with slight degradation as growth speeds increase to higher rates.
\end{abstract}

Keywords: A1. High resolution X-ray diffraction; A1. Cathodoluminescence; A3. Molecular beam epitaxy; B1. Gallium compounds; B1. Nitrides

\section{Introduction}

Over the past two decades gallium nitride has reached maturity as a technologically pivotal semiconductor. The uses for $\mathrm{GaN}$ range from power electronics, to high electron mobility transistors, and to light emitting diodes.[1,2] Currently, material for all of these applications has been predominantly produced by metal organic vapor phase epitaxy (MOVPE).

7 contamination, ability to make abrupt stops in incoming growth species (important for sharp quantum wells), and kinetically driven capability to access metastable phases that are not available to growth processes of thermodynamic equilibrium. However, any commercial interest in MBE based growth of $\mathrm{GaN}$ has been passed over due to slow growth rates that make it hard to meet demand thus challenging the economics of an MBE based industry. These slow growth rates are mostly due to the low fluxes of active nitrogen associated with conventional plasma nitrogen sources.[3]

The Energetic Neutral Atom Beam Lithography and Epitaxy (ENABLE) plasma source developed at Los Alamos National Laboratory (LANL) is a unique source. ENABLE is capable of delivering high flux of neutral nitrogen atoms (or other gases as well) as the active species to the surface of a wafer.[4] This technique has the potential to keep pace with the Ga fluxes supplied by the industry standard Ga effusion cells. This should lead to higher growth speeds for gallium nitride films by MBE. These high speed growth rates and their effects on structural and luminescent properties will be explored herein. 


\section{Experimental}

Film growth was performed in a custom built MBE chamber. This chamber exhibited a background pressure of $3.1 \times 10^{-9}$ Torr during the time prior to sample growth. Samples are thermally heated by radiative transfer from a pyrolytic boron nitride (PBN) heater plate $5 \mathrm{~mm}$ offset from the back of the wafer. Samples are rotated during growth at $5 \mathrm{rpm}$ to promote film uniformity. Reflection high energy electron diffraction (RHEED) is used to monitor the condition of the crystal surface. A residual gas analyzer (RGA) is used to measure partial pressures of relevant gases: $\mathrm{N}, \mathrm{NH}_{3}, \mathrm{H}_{2} \mathrm{O}, \mathrm{Ar}, \& \mathrm{CO}_{2}$. Transients of each signal are used to determine deviations from stoichiometry. A gallium beam is supplied to the sample surface of the sample from a Veeco SUMO thermal evaporator. This provides high quality Ga flux uniformity across the wafer surface. [5] A nitrogen beam is supplied to the sample from the LANL built ENABLE source.

The ENABLE source uses a focused infrared laser $(\lambda=10.6 \mu \mathrm{m})$ to sustain a plasma in an antechamber. Mass flow controllers are used to create a source gas mixture of $\mathrm{Ar}$ and $\mathrm{N}_{2}$. The flow rate of the $\mathrm{N}_{2}$ controls the flux of $\mathrm{N}$ atoms in the beam. The beam is created when the focused plasma is inserted into a supersonic nozzle. The expansion from this nozzle passes through several apertures and is introduced into the main growth chamber. This creates a beam of neutral nitrogen atoms with kinetic energies tunable from 0.5 to $2.0 \mathrm{eV}$.[6]

Samples were grown on two inches c-plane oriented $\alpha-\mathrm{Al}_{2} \mathrm{O}_{3}$ wafers. Wafers were outgassed for 30 minutes at $900^{\circ} \mathrm{C}$ followed by nitridation at $350{ }^{\circ} \mathrm{C}$ for 30 minutes. An aluminum nitride buffer layer was grown at $850{ }^{\circ} \mathrm{C}$ for 10 minutes at a nominal growth rate of 15 $\mathrm{nm} / \mathrm{min}$. Subsequently, the GaN layer was grown for 10 minutes at $850^{\circ} \mathrm{C}$ with varying growth rates. Growth rates were based on a nominal growth of $15 \mathrm{~nm} / \mathrm{min}$ for a Ga cell base temperature of $950^{\circ} \mathrm{C}$. Samples were also grown with the Ga cell base temperature at 1000 and $1050^{\circ} \mathrm{C}$. Under the assumption that the ratio of vapor pressures (based on Equation 1, where $\mathrm{T}$ is in Kelvin and $\mathrm{P}$ is in atmospheres [7]) at these temperatures will produce an equivalent ratio in $\mathrm{Ga}$ flux to the sample surface it was estimated that the growth rates should be $30 \mathrm{~nm} / \mathrm{min}$ and $60 \mathrm{~nm} / \mathrm{min}$, respectively. Growths were not investigated at higher speeds due to concerns of the Ga cell spitting above $1050^{\circ} \mathrm{C}$. However, it should be noted that the ENABLE source was only running at $20 \%$ nitrogen capacity for the fastest growth. In keeping consistency with other growth protocol on this tool (focused on In-rich InGaN [8]) films were grown in a slightly nitrogen rich regime. This was verified by monitoring the transient of the nitrogen signal in the RGA after metal shuttering.

\section{Equation 1: $\quad \ln \left(P_{G a, v a p}\right)=\frac{-14,900}{T}-0.515 \ln (T)+7.34$}

The thickness and surface roughness of the samples were measured using a J.A. Woollam variable angle spectroscopic ellipsometer. Incident light with wavelengths ranging from 400 to $800 \mathrm{~nm}$ was used. The resulting data was fitted to $n$ (index of refraction) and $\mathrm{k}$ (extinction coefficient) values for GaN and AlN. Surface roughness at the AlN-GaN interface was assumed to be negligible. This was based on the RHEED pattern of the AlN layer showing strong surface reconstruction, indicative of a near atomically flat surface.

Structural properties of the samples were characterized using a PANalytical MRD high resolution X-ray diffractometer (HRXRD). A hybrid X-ray mirror with asymmetrical Ge (220) 4-bounce crystal produces an incident $\mathrm{Cu}-\mathrm{K} \alpha_{1} \mathrm{X}$-ray beam with $\lambda=0.1540598 \mathrm{~nm}$ and 19 arcsec divergence. A triple crystal scheme (TC) utilizing a three-bounce Ge(220) analyzer crystal in front of the detector was used to collect high resolution $\omega-2 \theta$ and $\omega$ scans to separate coherent and diffused scattered radiation. A double crystal scheme (DC) with only a receiving slit on the 
detector was used to perform rough alignments and collect $\omega$ - $2 \theta$ and $\omega$-scans. A JEOL JSM 6300 scanning electron microscope (SEM) equipped with MonoCL2 cathodoluminescence spectrometer was used to measure the optical properties of the samples.

\section{Results and Interpretations}

Ellipsometry values for roughness and thickness are presented in Table 1. Most of these values track well with the X-ray diffraction based calculations. Both of the measured values trail the vapor pressure curve approximations. The offset in growth rates is due to the slight inaccuracy of the known growth rate. The reference growth rate was thought to be $15 \mathrm{~nm} / \mathrm{min}$, but these conditions yielded a growth rate of $13 \mathrm{~nm} / \mathrm{min}$.

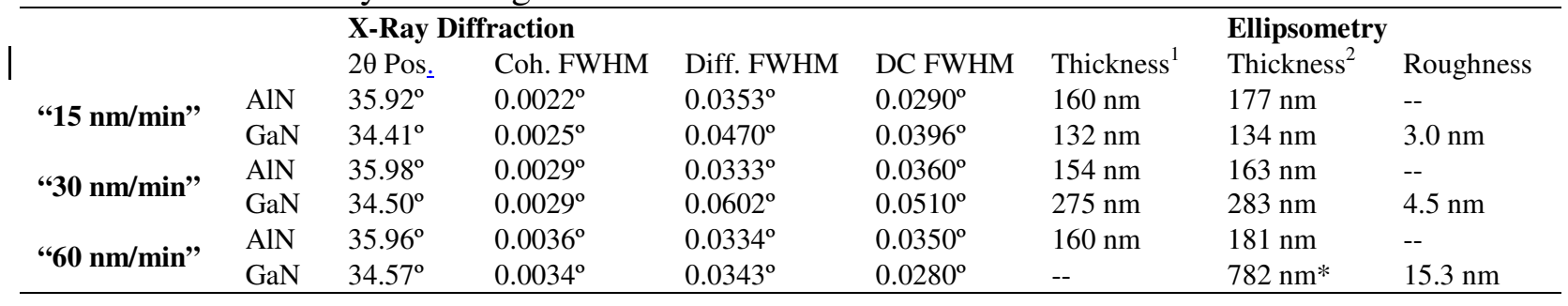

Table 1: Lists XRD and Ellipsometry measurements for the films. The "Coh. FWHM" column represents the full-widthhalf-maximum (FWHM) of a Gaussian peak representative of the narrow coherent peak for the TC $\omega$-scans. The "Diff. FWHM" represents the FWHM of the complimentary broad diffuse peak from the same de-convolution process of TC $\omega$ scan. The "DC FWHM" values are taken from $\omega$-scans with double crystal scheme and no receiving slits. Thicknesses 1 and 2 are from XRD and ellipsometry, respectively. *The thickness for the " $60 \mathrm{~nm} / \mathrm{min}$ " speed GaN does not follow the trend of the other two that is falling just short of the vapor pressure approximation. This value produced a high meansquared error and is likely off due to surface roughness and non-ideal $\mathbf{n} \& \mathbf{k}$ values.

A symmetric $\omega-2 \theta$ scan of all three samples is shown in Figure 1. The GaN (0002) and AlN (0002) can be clearly seen, with no irregular phases present. Additional scans (not shown here) included the $\mathrm{Al}_{2} \mathrm{O}_{3}$ (0006) substrate peak. These scans confirmed that measurements were made with the sapphire peak well aligned in the diffractometer at $2 \theta=41.67^{\circ}$. The high resolution TC $\omega-2 \theta$ scan resolves interference fringes (i.e. Pendellosüng fringes) about the main diffraction peaks. The angle spacing of the fringes can be used to estimate the layer thickness.[9] The fringes around the AlN peak correspond to a thickness of $\sim 160 \mathrm{~nm}$ for all three films, and hence a growth rate of $16 \mathrm{~nm} / \mathrm{min}$. The $\mathrm{GaN}$ peaks for the "15 $\mathrm{nm} / \mathrm{min}$ " and "30 $\mathrm{nm} / \mathrm{min}$ " both have fringes, while the " $60 \mathrm{~nm} / \mathrm{min}$ " does not due to higher volumetric structural deteriorations. The

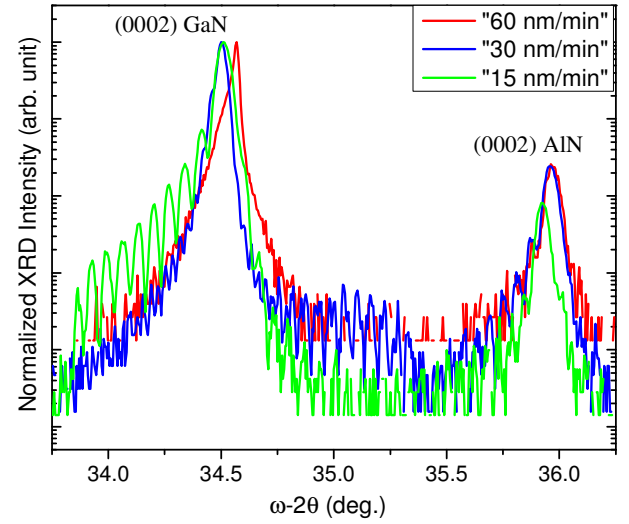

Figure 1: Semi-log plots of $\omega-2 \theta$ scans taken with the TC schematic, resolving the interference fringes on the main diffraction peak. Since these scans are symmetric the $\omega-2 \theta$ schematic is equivalent to $\theta-2 \theta$ scans. interference fringes on the former two correspond to thicknesses of 132 and $275 \mathrm{~nm}$, respectively. These values come from growth rates of 13.2 and $27.5 \mathrm{~nm} / \mathrm{min}$ for the nominal " $15 \mathrm{~nm} / \mathrm{min}$ " and "30nm/min" respectively. This is in close agreement with the initial vapor curve based approximations, and nearly identical agreement with the ellipsometry data. If it were to be assumed that the " $60 \mathrm{~nm} / \mathrm{min}$ " sample follows the same trend it would have a thickness of $550 \mathrm{~nm}$ and a growth rate of $55 \mathrm{~nm} / \mathrm{min}$. It is a noteworthy trend the Pendellosüng fringes 
become more diffuse with faster growth rates. This is likely due to increased bulk defect densities with increased growth rates, defects which may not show up in $\omega$-scans.
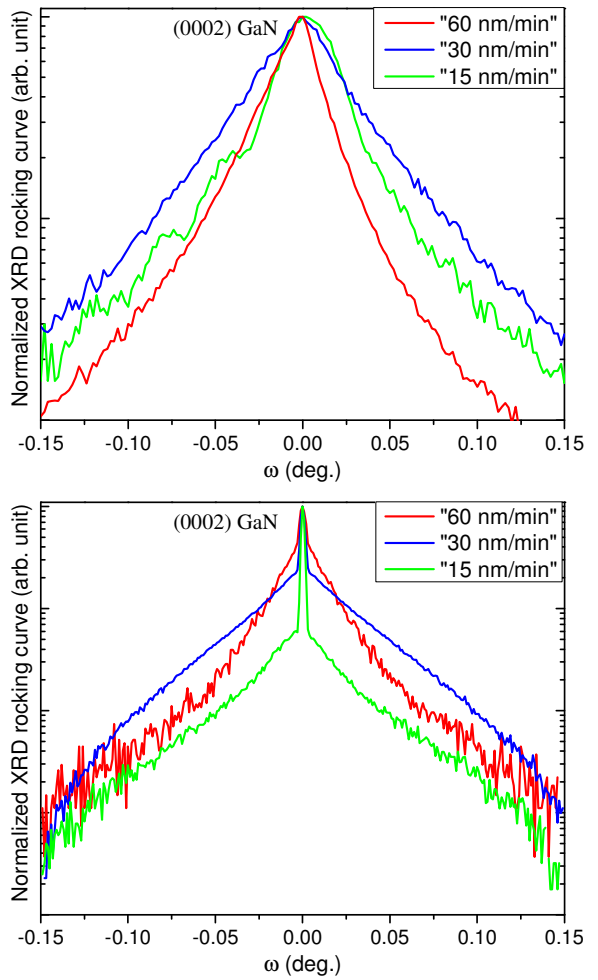

Figure 2a Depicts the GaN(0002) DC "no slit" $\omega$-scans of all samples. Peaks show a slight asymmetry. In the "15 $\mathrm{nm} / \mathrm{min} "$ sample this is due to interference fringes. Figure $2 b$ shows TC $\omega$-scans of the same peaks. Strong coherent peaks are flanked by wide diffuse peaks. The shape of the diffuse tails of the red RC suggest co-existence of two different types of crystal defects in the volume of GaN layer.
Figure 2 shows semi-log plots of $\omega$-scans about the $\mathrm{GaN}$ (0002) peak, taken with the double crystal and triple crystal schematic, respectively. The $\omega$-scan with the double crystal schematic was collected with no receiving slits. The triple crystal schematic uses the analyzer crystal to separate coherent diffraction of the lattice planes from diffuse radiation due to crystal imperfections. These scans have been de-convoluted into two Gaussian peaks representing the coherent and diffuse components. The FWHM for each of these component peaks is listed in Table 1. For the double crystal and diffuse scattered radiations the FWHM is never wider than $0.083^{\circ}$ or 300 arcsec. This value been classically assigned as benchmark device grade $\mathrm{GaN}$. There have been accounts that narrower peaks can lead to material with worse device quality.[10] Additional characterization of background carrier concentrations, TEM, and polarity measurements would indicate if this is valid for these samples. 
Cathodoluminescence (CL) spectra of the three samples, taken at $295 \mathrm{~K}$, are shown in Fig. 3(a). The "60 nm/min" sample exhibits a near-band-edge emission (NBE) at $364 \mathrm{~nm}$ with a FWHM of $11 \mathrm{~nm}$. Two additional broad emissions are observed at $\sim 450$ (blue) and $575 \mathrm{~nm}$ (yellow). The origin of the latter band is associated to nitrogen vacancies.[11] As the growth speed decreases, the intensity of the NBE and of the yellow band quench. A decrease in the yellow band luminescence may be associated to a decrease in the nitrogen vacancy density (i.e. material grows more stoichiometrically). CL images taken at the NBE emission of the three samples are shown in Figure 3. We observe an improvement in the film surface quality from large grains $(\sim 500 \mathrm{~nm})$ to smooth surfaces as the growth speed decreases. For example, Fig. 3("30 nm/min") exhibits bright and dark regions. The dark regions correspond to regions with lower $\mathrm{NBE}$ intensity, possibly due to lower crystalline quality. The decrease in the NBE for the lowest growth speed sample may be explained by a lower background carrier concentration.

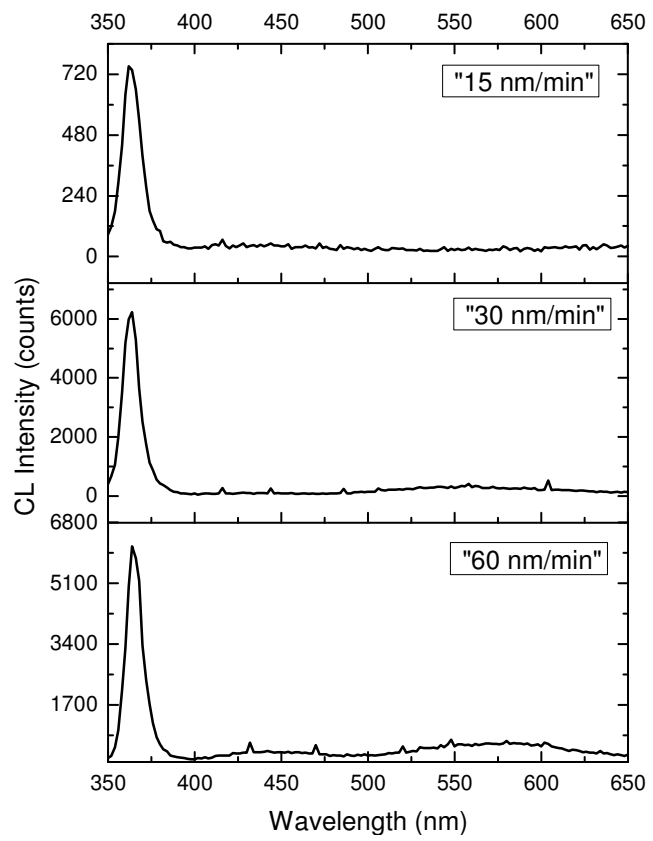

Figure 3 Cathodoluminescence spectra taken at room temperature. All spectra show band edge or near band edge emission. The " $15 \mathrm{~nm} / \mathrm{min}$ " sample has a significantly weaker intensity, likely due to a film thickness smaller than the excitation depth of the electron beam. The " $60 \mathrm{~nm} / \mathrm{min}$ " sample shows weak broad blue band and yellow band emission. Likely associated with point defects.

\section{Conclusion}

The use of the source technology ENABLE has been implemented in an MBE chamber to deliver high fluxes of active nitrogen for growth of gallium nitride. These high fluxes have been used to investigate nominal growth rates of 15, 30, and $60 \mathrm{~nm} / \mathrm{min}$. These films have been characterized by ellipsometry, x-ray diffraction and cathodoluminescence. Structures all show narrow FWHM for $\omega$-scans of (0002) GaN and interference fringes on the AlN buffer layer. The nominal 15 and $30 \mathrm{~nm} / \mathrm{min}$ films show interference fringes around the $\omega$ - $2 \theta \mathrm{GaN}$ (0002) peak, indicating high vertical coherence of these films. The $60 \mathrm{~nm} / \mathrm{min}$ film shows no interference fringes and broader almost diffuse $\omega$-scans, indicating that at high growth speeds the crystal quality is more deteriorated. This trend is also seen in the ellipsometry fittings. Both the ellipsometry fittings and the interference fringe spacing give good agreement for thicknesses in the "15 nm/min" and "30 nm/min" sample thicknesses. Real rates were slight slower than anticipated, 13 and $28 \mathrm{~nm} / \mathrm{min}$. However, the relative ratio for the rates was as expected.

All samples show band edge or near band edge emission in the cathodoluminescence measurements. This indicates that the growth rates can be pushed to high speeds while still sustaining crystal quality good enough for photo-active material. Some broad low intensity emissions in the blue and yellow range start to show up for the $60 \mathrm{~nm} / \mathrm{min}$ GaN, likely due to deviations in stoichiometry.

The characteristics of stable growth rate, narrow rocking curves, and photoactive material are all promising results. They indicate that using ENABLE as a source of active nitrogen species, nitride $\mathrm{MBE}$ can achieve growth respectable material at growth rates up to at least $55 \mathrm{~nm} / \mathrm{min}$. 
This rate of $3.3 \mu \mathrm{m} / \mathrm{hr}$ was achieved with the ENABLE source at $20 \%$ of maximum nitrogen capacity opening the possibility for MBE to achieve even faster nitride based growth.

Acknowledgments

This material is based upon work primarily supported by the Engineering Research Center Program of the National Science Foundation and the Office of Energy Efficiency and Renewable Energy of the Department of Energy under NSF Cooperative Agreement No. EEC-1041895. Any opinions, findings and conclusions or recommendations expressed in this material are those of the author(s) and do not necessarily reflect those of the National Science Foundation or Department of Energy. We gratefully acknowledge the use of facilities within the LeRoy Eyring Center for Solid State Science at Arizona State University.

\section{References}

[1] H. Morkoc, Nitride Semiconductors and Devices, Springer, Heidelberg, New York 1999.

[2] S. Nakamura, The Blue Laser Diode, Springer, 2000.

[3] N. Newman, The energetics of the GaN MBE reactions: A case study of meta-stable growth, J. Crystal Growth, 178 (1997) 102-112

[4] E. A. Akhadov, D. E. Read, A. H. Mueller, J. Murray, and M. A. Hoffbauer, Innovative approach to nanoscale device fabrication and low-temperature nitride film growth, J. Vac. Sci. Technol. B, 23 (2005) 3116.

[5] F. Ren, Proceedings of the Symposium on High Speed III-V Electronics for Wireless Applications and the Twenty-Fifth State-of-the-Art Program on Compound Semiconductors, The Electrochemical Society, Inc., Pennington, New Jersey, 1996.

[6] J.J. Williams, T.L. Williamson, M.A. Hoffbauer, Y. Wei, N.N. Faleev, and C.B. Honsberg, Growth of high crystal quality InN by ENABLE-MBE, Phys. Status Solidi C, 11 (2014) 577-580.

[7] C.N. Cochran, L.M. Foster, Vapor Pressure of Gallium, Stability of Gallium Suboxide Vapor, and Equilibria of Some Reactions Producing Gallium Suboxide Vapor, J. J. Electrochem. Soc., 109 (1962) 144-148.

[8] T.L. Williamson, A.L. Salazar, J.J. Williams, and M.A. Hoffbauer, Improvements in the compositional uniformity of In-rich $\operatorname{In}_{\mathrm{x}} \mathrm{Ga}_{1-\mathrm{x}} \mathrm{N}$ films grown at low temperatures by ENABLE, Phys. Status Solidi C, 8 (2011) 2098-2100.

[9] V. Holy, U. Pietsch, T. Baumbach, High resolution x-ray scattering from thin films and multilayers, Ch. 7, Springer Tracts in Modern Physics, V. 149, 1999.

[10] H. Morkoc, Nitride Semiconductors and Devices, Springer, Heidelberg, New York 1999, pp 124.

[11] E. R. Glaser, T. A. Kennedy, J. A. Freitas, Jr., M. Asif Khan, D. T. Olsen, and J. N. Kuznia, Silicon Carbide and Related Materials, IOP Conf. Proc. No. 137 (IOP, Bristol, 1994), p. 443. 